\title{
The ring galaxy HRG 54 103: a first study ${ }^{\star}$
}

\author{
M. Faúndez-Abans ${ }^{1}$, I. F. Fernandes ${ }^{1}$, M. de Oliveira-Abans ${ }^{1}$, P. C. R. Poppe ${ }^{2}$, and V. A. F. Martin ${ }^{2}$ \\ 1 MCT/Laboratório Nacional de Astrofísica, Rua Estados Unidos 154, Bairro das Nações, 37.504-364 Itajubá, MG, Brazil \\ e-mail: [mfaundez; ifernandes; mabans] @lna.br \\ 2 Universidade Estadual de Feira de Santana, Departamento de Física e Observatório Astronômico Antares, BA, Brazil \\ e-mail: [paulopoppe;vmartin1963]@gmail.com
}

Received 26 September 2008 / Accepted 27 August 2009

\begin{abstract}
Aims. We report the first study of the peculiar ring galaxy HRG 54103 which was previously classified as a Saturn-like type galaxy. Methods. The study is based on low resolution spectroscopy and photometric observations in the optical band to highlight the characteristics of this almost isolated galaxy. The colour distribution of HRG 54103 was examined through direct CCD BVRI Kron-Cousins system imagery. Color-color diagrams of the bulge and ring are displayed and further compared with the star-forming ring galaxy HRG 2302.

Results. The results of image enhancement of the morphological structure of this galaxy are discussed. The nuclear emission-line spectrum resembles that of a Seyfert2/LINER object, with $z=0.022$ and heliocentric $V=6483 \pm 18 \mathrm{~km} \mathrm{~s}^{-1}$, in agreement with the literature. The nuclear, bulge and ring section radial velocities along the ring major axis show a peculiar distribution, which together with the $[\mathrm{N}$ II] $/ \mathrm{H} \alpha$ and $[\mathrm{S} \mathrm{II}] / \mathrm{H} \alpha$ ratios and image enhancement suggest an offset nucleus and an internal tilted ring or shell.

Conclusions. HRG 54103 is a peculiar galaxy with an intermediate activity region, probably due to a residual excitation effect through the central AGN phenomenon. The individual sections of the color-color map are redder than a typical star-forming ring galaxy, in agreement with the behavior of the $[\mathrm{S}$ II] $/ \mathrm{H} \alpha$ versus $[\mathrm{N}$ II $] / \mathrm{H} \alpha$ diagnostic diagram. The two bulge satellites, the plume-like appendix, and the disk-ring asymmetry suggest a possible merger event in the recent past of this object, which could also have caused its formation.
\end{abstract}

Key words. galaxies: Seyfert - galaxies: photometry - galaxies: kinematics and dynamics - galaxies: individual: HRG 54103 galaxies: active - galaxies: peculiar

\section{Introduction}

Gravity is the acting force that makes galaxies interact, collide, and merge. Colliding galaxies are twisted and deformed by their mutual gravitational fields. This often gives galaxies peculiar shapes and can create tails, plumes and bridges between the interacting objects. These features are simply the patterns of the gas, dust and some stars which have been drawn out by tidal forces during the interaction. Also, interactions are seen in galaxies that form rings in their life time-line. An intruder galaxy can plunge through the center (or close to it) of a rotating disk galaxy, triggering the birth of bright young stars in the wake of radially expanding annular shock waves. Those objects are called ring galaxies (RGs) and belong to the morphological category 6 of the "Catalogue of Southern Peculiar Galaxies and Associations" (see Arp \& Madore 1977, 1986, as a starting point of the study of peculiar galaxies with rings). In general, the rings may either be collisional (peculiar - pRG) or resonant (normal NRG, see Faúndez-Abans \& de Oliveira-Abans 1998a, for this denomination). It is well accepted that collisional RGs are important regions to study disks that remain fairly intact after the interaction, allowing the observation of its structures and star formation properties in detail, such as galaxy-scale perturbation

^ Based on observations made at: (a) Observatório do Pico dos Dias, operated by MCT/Laboratório Nacional de Astrofísica, Brazil, and (b) Cerro Tololo Inter-American Observatory, National Optical Astronomy Observatories, which are operated by AURA, Inc., under contract to the National Science Foundation. properties, hydrodynamics, vigorous non-nuclear star formation and stellar evolutionary processes across and inside the ring.

The earliest discussions on RGs are found in the works of Burbidge \& Burbidge (1959) and Freeman \& de Vaucouleurs (1974) suggesting the two types of objects that collide to form rings. The basic observational properties of a sample of RGs were determined in the 70s by Theys \& Spiegel $(1976,1977)$ and their earliest numerical simulation regarding their origin were carried out by Lynds \& Toomre (1976). Multiple-band observations were performed by Appleton \& Struck-Marcell (1997, and references therein). The prototype RG, the Cartwheel, has been tested observationally to study the ring expansion, star formation in the ring, radial color gradient and stellar ages (see Fosbury \& Hawarden 1977; Higdon 1995; Marcum et al. 1992; Korchagin et al. 2001; Vorobyov \& Bizyaev 2001). Color gradients were found in some other RGs (Appleton \& Marston 1997), and Marston \& Appleton (1995) found that the majority of the star-forming regions are located in the ring. Recently, some results from GALEX and SPITZER surveys of a dozen collisional RGs have been used by Appleton et al. (2008), who have investigated massive star formation in the ring, with interesting results about the Cartwheel ring wave propagation and the type of luminous sources in the ring.

In the last four decades, a number of classification systems and studies of some individual cases have been put forward. The ring galaxies classification suggested by Theys \& Spiegel (1976) includes only objects that show crisp, elliptical and knotted rings, rings without a nucleus, or with an off-center nucleus 


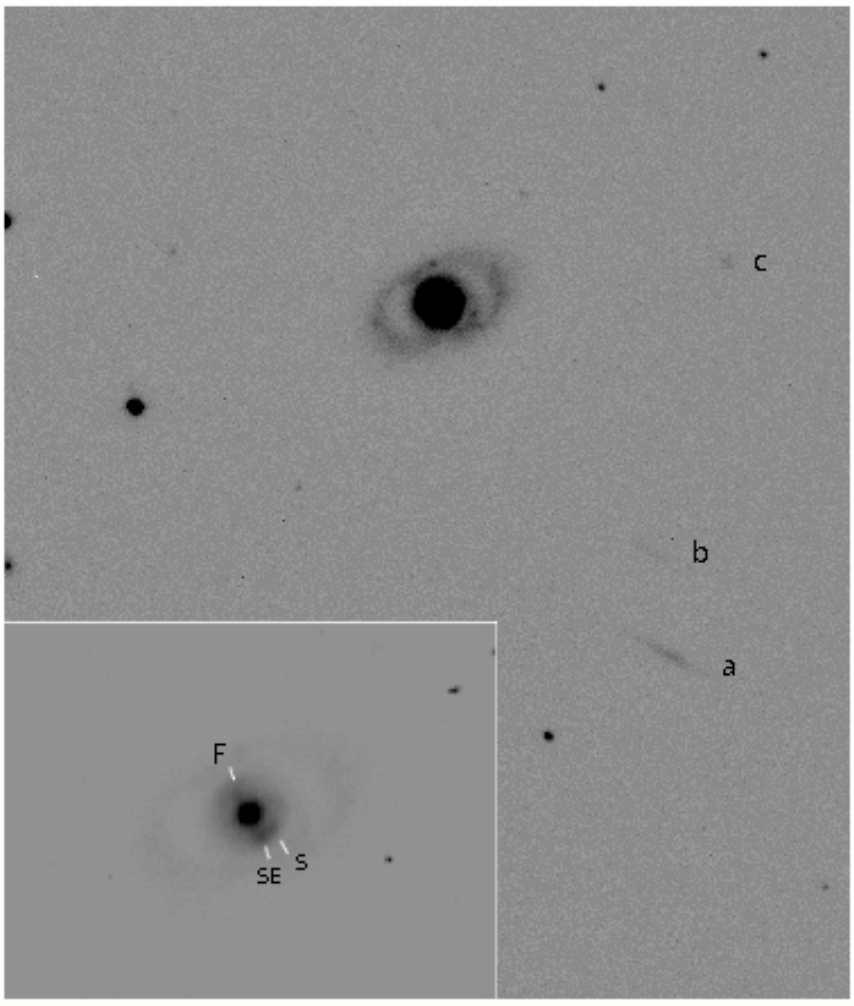

Fig. 1. The Saturn-like galaxy HRG 54103. Original 3.' $5 \times 4 .{ }^{\prime} 3 B$ frame obtained at OPD. North is up, east is on the right. Two new galaxies not previously catalogued, seen here almost edge-on, have been found in this field on the SE direction and named $a$ and $b$. There is a third object, c, in the E direction. The inserted figure shows the two Southern substructures SE and S, and the Northern plume-like appendix, F. The substructures are placed at almost 2". 1 South and South-East, and 2". 1 North from the center of the object, respectively, on the inner ring - see Table 4.

- all objects with a strongly perturbed life history. This family of objects corresponds to the P-type rings proposed by Few \& Madore (1986), who proposed the existence of another broad class of rings named O-type, which have a smooth structure and a centrally-located nucleus, in general without companions. Within this O-type family of objects, lenticular galaxies with purely stellar rings have been observed to show structures that resemble Saturn and its rings. A prototype of such objects is ESO 192-G09, a galaxy without any conspicuous companions, which shows a smooth inner ring surrounding a spheroidal bulge and with no signs of a bar. Outside this inner ring, a much fainter ring is present and seems to be connected to the inner regions at two diametrically opposite points, in a manner very much like that of early-type outer-ringed barred galaxies (Buta 1984). This object has been classified as SA(r) $0^{+}$by Buta (1995), but an inspection of the SRC/ESO J and R films reveals that ESO 192-G09 looks like either a ringed S0 or a flattened bulge Saturn-like object. A better example of the Saturn-like pRG category is the galaxy HRG 54103, as easily seen in the SRC/ESO film archives. This object was pointed out as one of the best archetypes of Saturn-like objects by Faúndez-Abans \& de Oliveira-Abans (1998a, hereafter FAOA, in a Peculiar Ring Galaxies-pRG-classification).

In this work, we focus on the morphologically Saturn-like pRG, the galaxy HRG 54103 (Fig. 1), which looks like Saturn in observations of the ESO/UPPSALA survey in the ESO(B) atlas (Faúndez-Abans et al. 1992; see also NED for more references) and has been classified as type $\mathrm{LAR}+(\mathrm{RC} 3)$ and $\mathrm{SA}(\mathrm{r}) 0^{+}$by Buta (1995). On the other hand, it looks like an isolated galaxy, and this first insight will contribute to our knowledge of this type of galaxy in the near Universe, with the future idea to compare its characteristics with other RGs like GDS J033206.44274728.8 (Dominici 2009, an object with $z \approx 1.02$ ). The internal structure of HRG 54103 was studied for the first time by Faúndez-Abans \& de Oliveira-Abans (1998b, hereafter FA98b), who highlighted some fine structure as shown in Figs. 11 and 16 of that work: two round satellites in the periphery of the bulge, and a short plume (SE, S and F in Fig. 1 insert, respectively). Those enhanced features could be the result of an orbital resonance in the galactic field, forming a warped ring. A few extragalactic objects appear in the observed field of HRG 54103, the most prominent being two edge-on disk galaxies in the SE direction (see Fig. 1). The coordinates of their centroids (J2000), as calculated differentially from the centroid of HRG 54103, are: $\alpha=01^{\mathrm{h}} 18^{\mathrm{m}} 48^{\mathrm{m}} \cdot 48, \delta=-19^{\circ} 39^{\prime} 04^{\prime \prime} .85$ for the larger galaxy, with no reported redshift in the literature, named APMUKS(BJ) B011622.67-195450.6 in the APM galaxy survey by Maddox et al. (1990), and $\alpha=01^{\mathrm{h}} 18^{\mathrm{m}} 48^{\mathrm{m}} \cdot 10, \delta=-19^{\circ} 38^{\prime} 38^{\prime \prime}$. 00 for the smaller one, both without redshift determinations in the literature (see Fig. 1: a and b, respectively). These coordinates are expected to be accurate to $1^{\prime \prime}$ each. There are two other references about this galaxy in the literature. In one, it is listed as target of an image database by Garnier et al. (1996, Monograph of the Extragalactic Database). The other one is the first redshift determination by da Costa et al. (1998) in a Southern redshift survey. No prominent extragalactic objects are quoted by NED within 5.0 arcmin around HRG 54103.

Details of our observations and data reduction are displayed in Sect. 2. The obtained results are discussed in Sect. 3. The conclusions of this study are presented in Sect. 4.

\section{Observations and data reduction}

An observational campaign was carried out at two observatories: (1) at the Observatório do Pico dos Dias (OPD) - Laboratório Nacional de Astrofísica, Brazil, on the 1.6-m telescope using the direct CCD camera with $B V R I$ Kron-Cousins filters (Bessel 1990 ) and a $384 \times 578$-pixel chip with a scale of 0.284 arcsec pix ${ }^{-1}\left(0.128 \mathrm{kpc} \mathrm{pix}^{-1}\right.$ and $\left.0.451 \mathrm{kpc} \operatorname{arcsec}^{-1}\right)$; three frames in each filter with individual exposure times of $600 \mathrm{~s}$ were taken under photometric conditions better than 1".2 FWHM seeing; the stars used for extinction and calibration are from Landolt (1992); (2) spectroscopic observations performed at CTIO (Cerro Tololo Inter-American Observatory) with the $1.5-\mathrm{m}$ telescope equipped with a Cassegrain spectrograph and Loral $1 \mathrm{k} \# 1+$ Arcon $3.9(1200 \times 800$ square pixels, pixel size of $15 \mu \mathrm{m}$, scale of $\left.1.3^{\prime \prime} \mathrm{pix}^{-1}\right)$. The grating \# 35 of $6001 \mathrm{~mm}^{-1}$ was centered at $675 \mathrm{~nm}$. Two spectra (20 minutes each) were obtained. The $3^{\prime \prime}-$ slit was aligned exactly with the major ring axis of the object $\left(\mathrm{PA}=61^{\circ}\right)$, close to the SW-NE direction. The stars used for extinction and flux calibrations are tertiary standards from Baldwin \& Stone (1984), as revised by Hamuy et al. (1992, see also Hamuy et al. 1994).

The CCD image processing and data analysis have been done using standard procedures, IRAF and STSDAS packages. Each image has a small spatial dithering. All the HRG 54103 frames were aligned using four isolated stars in each frame, then collapsed for each filter using IMCOMBINE, which also allows the removal of the cosmic rays, as a preliminary task (a second run of the IRAF COSMIC RAY task was made afterwards). Almost 22 photometric standard stars from Landolt (1992) were observed 
and the calibration to the standard $B V R I$ photometric system was performed in the usual way (see e.g. Reshetnikov et al. 1994). The standard deviation $\sigma$, for the calibration to the $B V R I$ filters were $0.059,0.037,0.042$ and 0.056 , respectively. The sky brightness, in mag $\operatorname{arcsec}^{-2}$ for the filters, were 21.70, 20,51, 19.80 and 18.30 , respectively. The PSF was determined based on 14 isolated stars in each frame. The observed field of HRG 54103 lacks reasonably bright stars for this calculation. The removal of foreground stars was achieved with IRAF/PEAK/SUBSTAR. The sky background was estimated by examining the median count in concentric rings of different widths centered on the galaxy with an external radius extending to the limits of the frame. The integrated photometry was performed with the STSDAS/ELLIPSE and IRAF/POLYPHOT, respectively. The background level uncertainty was $1.0-1.1$.

The spectra processing and data analysis were done using standard procedures employing IRAF and RVSAO packages. This includes bias subtraction, flat field correction, cosmic ray removal, sky subtraction, wavelength and flux calibration (IMAGES/IMFIT; IMUTIL, STSDAS/IMGTOOLS, TWODSPEC and ONEDSPEC tasks, respectively). The useful spectral wavelength range was $\lambda \lambda 5750-7200 \AA$ and the wavelength calibration error was $\simeq 10 \AA$. The standard extraction aperture was set for the emission region. The spectra were reduced to a scale flux per unit wavelength using the measurements of the standard stars observed at similar airmasses. The line fluxes were obtained using the IRAF/SPLOT task. This task was also used to obtain the center of the emission lines in order to later calculate the radial velocities of the measured lines. As a double check of these results, the RVSAO/IRAF external package was used to calculate the apparent radial velocities from the observed spectral shifts. The EMSAO task finds emission lines in a spectrum and computes the observed centers, yielding individual shifts and errors for each line as well as a single velocity by combining all of the lines (Mink and Wyatt 1995). A value of $H_{\mathrm{o}}=70 \mathrm{~km} \mathrm{~s}^{-1} \mathrm{Mpc}^{-1}$ was adopted throughout this work.

\section{Analysis and results}

FA98b studied the morphology of this object looking for internal structure and found a few substructures (see Figs. 11 and 16 of that work). In the present work, we took several measurements on these substructures (the Northern filament, F; the two Southern knots, SE and S, highlighted in the insert of Fig. 1); the sizes of the nucleus, bulge and ring, the new results of velocity and $z$, together with some early information on HRG 54103, are listed in Table 1.

\subsection{Surface photometry}

In an early work, FA98b studied some morphological features in two-dimensional images of HRG 54103 which they enhanced through application of a transform function on the spatial frequency domain and further processing of the images. In that work, Figs. 11 and 16 show the presence of three apparent satellites (two spherical and a filament-like one).

To perform the integrated photometric measurements, isophotal analysis was done using the STSDAS task ELLIPSE, and the IRAF task POLYPHOT. Table 2 displays the BVRI magnitudes and integrated colors within the 25-mag-per-squarearcsec ellipse, and the integrated surface brightness in the central $10^{\prime \prime}$-diameter circle. Our $B$ results are in agreement with those of the ESO/Uppsala Survey of the ESO(B) Atlas (Lauberts 1982).
Table 1. Data on HRG 54103.

\begin{tabular}{|c|c|c|}
\hline Parameter & Value & Ref. \\
\hline RA (2000) & 011844.36 & FAOA \\
\hline Dec. (2000) & -193736.33 & FAOA \\
\hline Hubble type & $\mathrm{SA}(\mathrm{r}) 0^{+}$ & Buta (1995) \\
\hline \multirow[t]{4}{*}{ Other designations } & ESO 542-G03 & NED \\
\hline & MCG-03-04-042 & NED \\
\hline & IRAS 01162-1953 & NED \\
\hline & PGC 004703 & NED \\
\hline pRG-family & Polar/Saturn-like & FAOA \\
\hline$z$ & 0.0213 & NED \\
\hline$z_{\text {emiss }}$ & 0.022 & this work \\
\hline$V\left(\mathrm{~km} \mathrm{~s}^{-1}\right)$ & 6396 & NED \\
\hline$V_{\text {emiss }}$ & $6483 \pm 18$ & this work \\
\hline Mass & $7.24 \times 10^{10} M_{\odot}$ & this work \\
\hline Nucleus major axis $(R)$ & $2 ! .048$ & this work \\
\hline Nucleus minor axis $(R)$ & 1.. 607 & this work \\
\hline Bulge major axis $(R)$ & 5.75 & this work \\
\hline Bulge minor axis $(R)$ & 5.' 60 & this work \\
\hline Ring major axis $(R)$ & $177^{\prime \prime} 02$ & this work \\
\hline Ring minor axis $(R)$ & 10.96 & this work \\
\hline $\mathrm{N}$ filament length $(R)$ & $1 " .025$ & this work \\
\hline $\mathrm{S}$ knots diameters $(R)$ & 0.4 & this work \\
\hline Distance & $93 \mathrm{Mpc}$ & this work \\
\hline
\end{tabular}

$R$ : Measurements on the $R$-filter image.

Table 2. Integrated photometric data.

\begin{tabular}{lr}
\hline \hline Parameter & $\begin{array}{r}\text { Value } \\
(\mathrm{mag})\end{array}$ \\
\hline$B_{25}$ & $14.25 \pm 0.10$ \\
$V_{25}$ & $13.34 \pm 0.10$ \\
$R_{25}$ & $12.80 \pm 0.10$ \\
$I_{25}$ & $12.28 \pm 0.10$ \\
$(B-V)_{25}$ & $0.91 \pm 0.15$ \\
$(B-R)_{25}$ & $1.46 \pm 0.15$ \\
$(V-R)_{25}$ & $0.55 \pm 0.15$ \\
$(R-I)_{25}$ & $0.51 \pm 0.15$ \\
$(V-I)_{25}$ & $1.06 \pm 0.15$ \\
$(B-I)_{25}$ & $1.97 \pm 0.15$ \\
$B_{10}$ & $20.29 \pm 0.09$ \\
$V_{10}$ & $19.44 \pm 0.09$ \\
$R_{10}$ & $18.87 \pm 0.09$ \\
$I_{10}$ & $18.34 \pm 0.09$ \\
\hline
\end{tabular}

10: Mean integrated surface brightness for $10^{\prime \prime}$ aperture.

Table 3. The $B-R$ and $B-V$ values of the spherical structures and filament.

\begin{tabular}{lcc}
\hline \hline Structure & $\begin{array}{l}B-R \\
(\mathrm{mag})\end{array}$ & $\begin{array}{l}B-V \\
(\mathrm{mag})\end{array}$ \\
\hline S sphere-like & 2.913 & 1.829 \\
SE sphere-like & 2.694 & 1.636 \\
Filament & 2.839 & 1.672 \\
\hline
\end{tabular}

Table 3 displays the $B-R$ and $B-V$ colors of the Southern substructures $\mathrm{S}$ and $\mathrm{S}-\mathrm{E}$, and the Northern filament N, located, respectively, at about 2".1 South and South-East, and 2".1 North, of the center of the object (see the insert in Fig. 1). These structures are redder than the other galaxy components, suggesting the presence of an older population and/or of a reddening excess on both the NW and SE sides of HRG 54103, close to the bulge.

To perform photometric measurements on selected regions of the bulge and of the ring, a diaphragm of $4 \times 4$ pix $^{2}$ was 


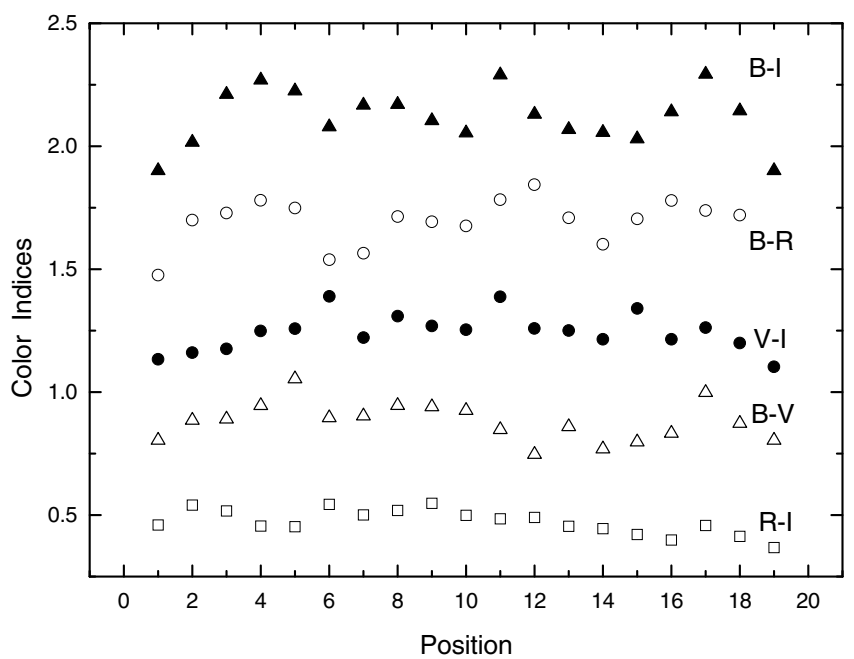

Fig. 2. Color indices versus arbitrary positions in the ring of HRG 54103. The errors are: \pm 0.18 in $B-I, \pm 0.15$ in $B-R, \pm 0.10$ in $V-I, \pm 0.15$ in $B-V$ and \pm 0.09 in $R-I$. The measurements along the ring were made in the clockwise direction from the North, from $\mathrm{PA}=318^{\circ} \rightarrow 0^{\circ} \rightarrow 80^{\circ}$, ending at $\rightarrow 318^{\circ}$ ).

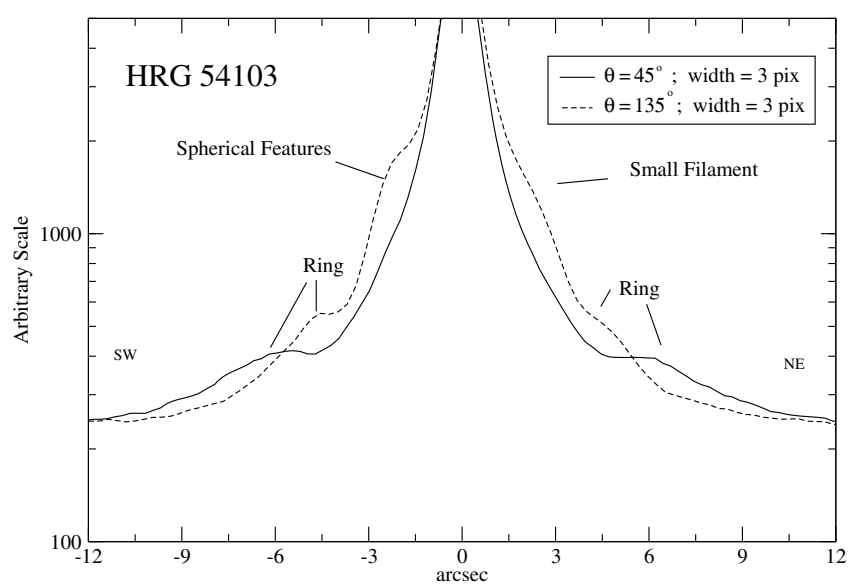

Fig. 3. Brightness profile on an arbitrary scale after image enhancement (FA98b). The continuous line is the profile along the ring major axis, and the segmented line is the profile along the nuclear major axis (the galaxy minor axis), which encompasses the substructures that lie along this direction (almost close to the ring minor axis). Those structures are recognized and indicated in the figure.

used. The measurements did not cover all the projected surface of HRG 54103, but all the brightest structures were studied. The measurements along the ring were made in the clockwise direction from the North, covering all the ring. Figure 2 displays the color indices for 19 apertures. In this figure, we see that in $B-R$ there are three bluer regions which correspond to the brighter clumps on the ring (positions 1, 6-7, and 14), suggesting that those may be star-forming regions. Color-color diagrams highlight bluer and redder regions in galaxies. Appleton \& Marston (1997) have shown that, in diagrams of $B-R$ versus radial distance, there is a clear drop in the values of the color index where blue star-forming regions are present on the ring. This $B-R$ index then seems to be an indicator of possible star formation.

HRG 54103 is almost face-on and its brightness profiles along both principal axes are displayed in Fig. 3 in two slices of $3 \times 150$ pixels on the $\mathrm{R}$ image. One was chosen to lie along the major axis of the ring $\left(\theta=45^{\circ}\right.$, measured anti-clockwise from the $\mathrm{E}$ to $\mathrm{W}$ ), and the other along the major axis of the

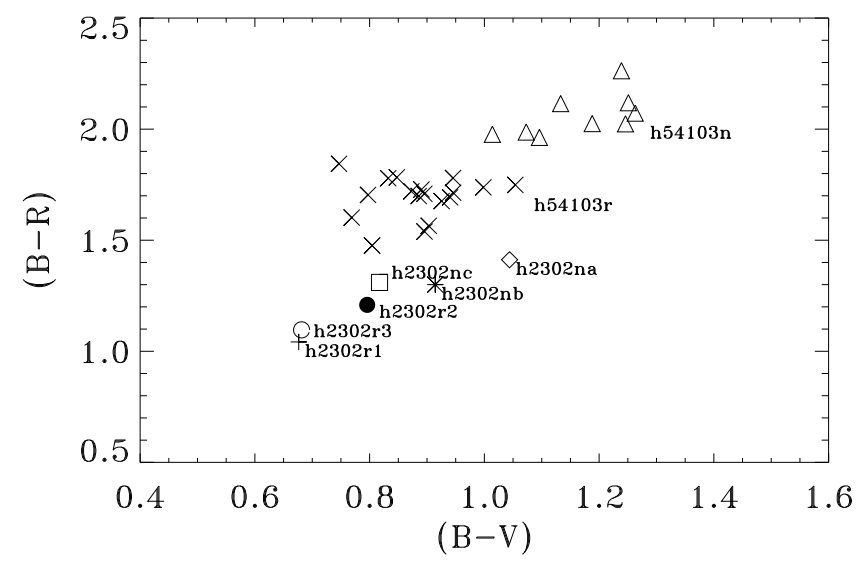

Fig. 4. $B-R$ versus $B-V$ diagram. Each data point is the result of measurements within diaphragms of $4 \times 4$ pixels. The symbols: (1) for HRG 54103, "n" $(\triangle)$ and "r" $(\times)$ stand for bulge and the ring, respectively; (2) for HRG 2302, "na" ( $\diamond)$ and "nb" $(*)$ represent the I and II regions displayed in Fig. 9 of MVFOS (both nuclear structures); "nc" (口) the bulge-like knot, region III in the same figure (a prominent ring component), and $\mathrm{r} 1(+), \mathrm{r} 2(\bullet)$ and $\mathrm{r} 3(\bigcirc)$ stand for $(1,7,2),(3,4)$ and $(5$, 6)in the same figure, respectively.

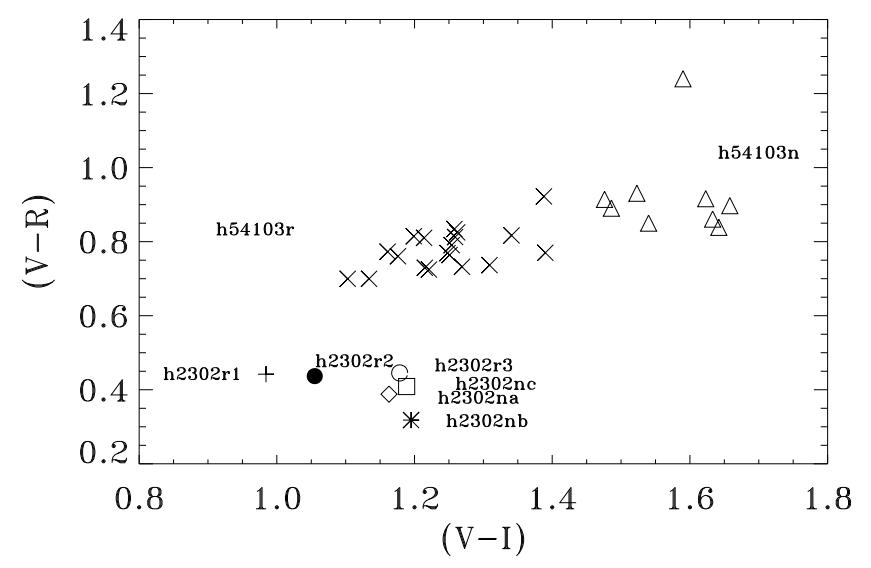

Fig. 5. The same as Fig. 3 for $V-R$ versus $V-I$.

central body $\left(\theta=145^{\circ}\right)$, which crosses the Northern filament and is close to the middle point of the two "spherical" bodies to the South.

The inspection of this figure yields the following: (1) the ring major axis profile has an almost symmetrical light distribution across the bulge and nucleus; (2) the ring's light contribution is apparent as bumps both on the SW and NE sides; (3) the brightness profile of the nuclear major axis is asymmetrical on both sides of the ring connection with the bulge; (4) the spherical and filamentary bodies' contribution are noticeable on the profile (dashed line).

\subsection{Color-color diagrams}

In FA98b, HRG54103 did not show strong visual evidence of star-forming regions in the ring, in spite of the fact that some regions, when highlighted, display condensation of material (clumps). Are we looking at a purely stellar ring plus H II knots which may be the beginning of future $\mathrm{H}$ II regions? In view of this supposition, we compare the behavior of this object with a star-forming ring galaxy in color-color diagrams.

Figures 4-7 show the color distribution of selected regions of HRG 54103. In these color-color diagrams, we have adopted the 


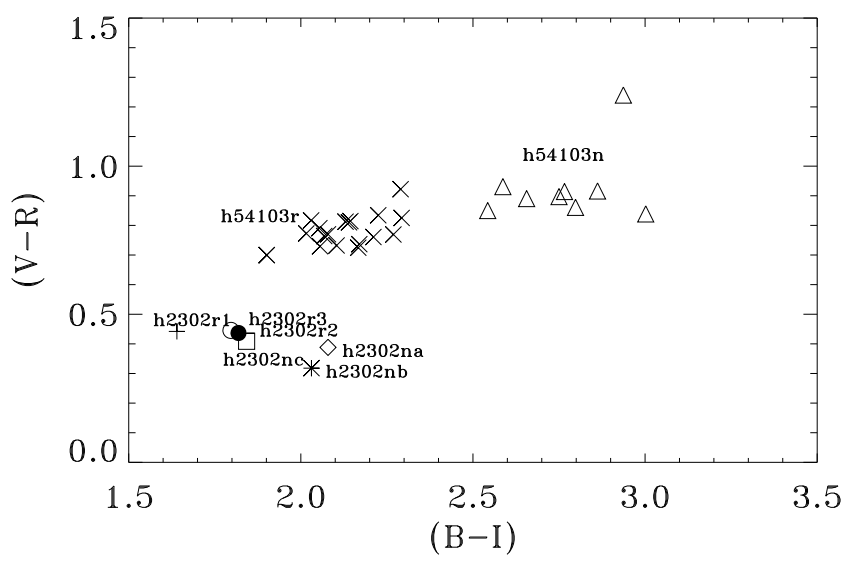

Fig. 6. The same as Fig. 3 for $V-R$ versus $B-I$.

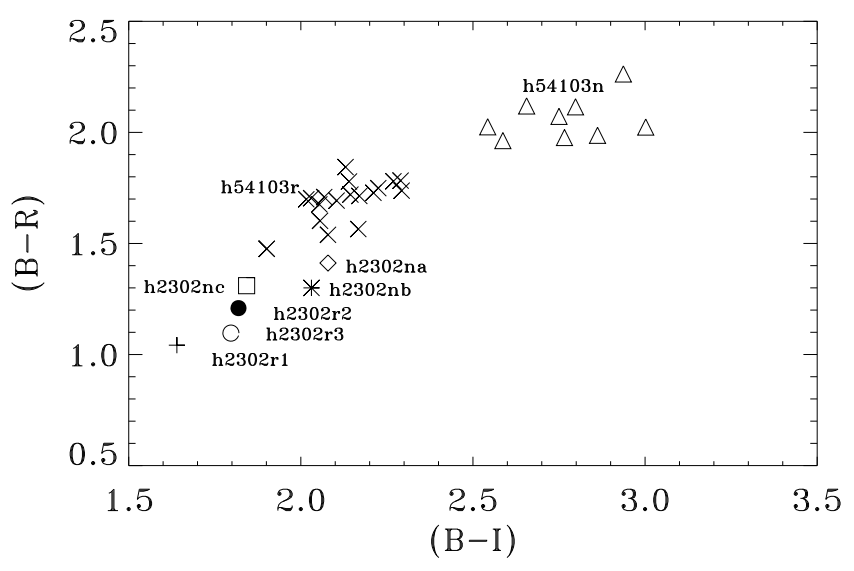

Fig. 7. The same as Fig. 3 for $B-R$ versus $B-I$.

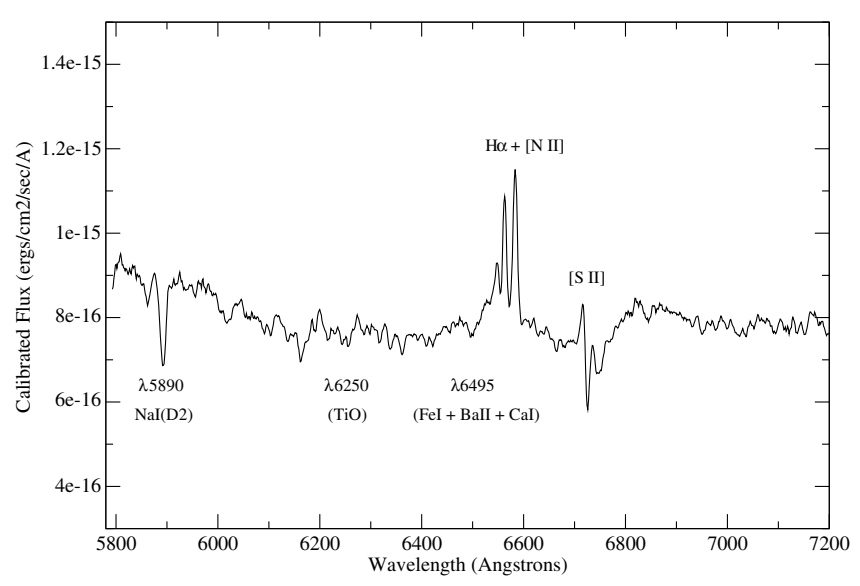

Fig. 8. The nuclear features of HRG54103 - see main text for discussion.

star-forming ring galaxy HRG 2302 (Myrrha et al. 1999, hereafter MVFOS) as a standard with which to compare HRG 54103. These galaxies present similar redshift values $(0.02$ and 0.022 respectively), and the color values were determined with the same telescope, instrumental setup, and standard group of stars. One difference between them is that HRG 2302 has H II regions and a confirmed companion in the field, while HRG 54103 has no evident companion galaxy.

Comparing HRG 54103's results of the present work with HRG 2302's and other collisional-rings with star forming knots - e.g. Appleton \& Marston (1997), and Bransford et al. (1998)
- we see that in the diagram of Fig. 4, the $B-V$ color distribution for both galaxies are consistent at $0.65 \leq B-V \leq 1.2$ for HRG 2302 and $0.7 \leq B-V \leq 1.25$ for HRG 54103, while the $B-R$ colors in HRG 54103 are redder than HRG 2302: $-1.5 \leq B-R \leq 2.3$ and $-1.0 \leq B-R \leq 1.5$, respectively. This is also valid for the color-color plots of Figs. 5-7. These figures also show a separation of the objects, HRG 2302 being "bluer" than HRG 54103. It is also possible to distinguish the distribution of data points of the ring from the bulge in HRG 54103. In Figs. 4-7, the upper triangle $(\triangle)$ represents the nucleus of HRG 54103.

The distribution of the test color data points in HRG 2302 shows that the central regions such as the nuclear (na) and the tail-like (nb) are redder. Also, the "bulge-like" prominent knot (nc - the probable displaced nucleus of the target galaxy) is redder than the knots of the ring (e.g. r1 to r2 in Fig. 4). This suggests a contribution of an old population at the center and nb region, and probably star forming regions in the knotty ring (see the simplified sketch of Fig. 9 in MVFOS).

\subsection{Spectra}

The CTIO spectra ( $\lambda \lambda 5800-7200 \AA$ region, see Fig. 8) were obtained with the slit aligned with the ring major axis, almost along the SW-NE direction, encompassing the SW ring knot and the fainter knot and rips of the NE ring section. The following lines can be discerned from left to right: the absorption NaID line, the $[\mathrm{N} \mathrm{II}] \lambda 6548, \mathrm{H} \alpha,[\mathrm{N} \mathrm{II}] \lambda 6583$, and [S II] $\lambda 6716$ lines and the atmospheric $\mathrm{O}_{2}$ band.

The nuclear section of the spectra show emission lines characteristic of AGN-like objects. In this region, the [N II] $\lambda 6583$ line is stronger than $\mathrm{H} \alpha$, but there are no [O I] $\lambda 6300$ lines in the spectrum. We suggest a Seyfert2/LINER nuclear type, instead (see Kennicutt et al. 1996). The spectra (see Fig. 8) resemble, in part, the spectra of NGC 4941 (Seyfert 2) and NGC 4579 (LINER), superposed on a normal galaxy spectrum, such as NGC 3368 (see references quoted in NED for these galaxies). The absorption features in the spectrum of HRG 54103 (in the $\lambda \lambda 5800-7200 \AA$ region) suggest the contribution of intermediate-mass stars (see Origlia et al. 1999; Guzeva et al. 2000; Fernandes et al. 2004). The spectra of the ring sections exhibit star forming characteristics.

There are two possible explanations for the excess of [N II] $\lambda 6584$ emission in the spectra of HRG 54103 (see Fig. 8): (a) the first one applies to starburst and H II galaxies, which have an overabundance of nitrogen with respect to normal H II regions (Stauffer 1982); such overabundances have been reported by Gonzalez-Delgado et al. (1995) and the explanation for this, as proposed by Lehnert \& Heckman (1994), is that shocks produced by starburst winds or supernovae could probably cause this; (b) the second one is that HRG 54103 has an extra source of gas excitation, in addition to the AGN phenomenon as some works have suggested (Storchi-Bergmann 1991; StorchiBergmann \& Wilson 1996; Ohyama et al. 1997); this is the preferred solution of Kennicutt et al. (1989), who suggested hidden active nuclei as the extra source.

Figure 9a displays the rotation curve of the central region, bulge and ring sections of HRG 54103. For the calculation of the velocity, we have considered the values corresponding to the maxima of the continuum intensity as systemic velocities. The derived heliocentric systemic velocity for HRG 54103 is $6483 \pm 18 \mathrm{~km} \mathrm{~s}^{-1}(z=0.022)$, which is in agreement with NED's released value (see Table 1$)$. 

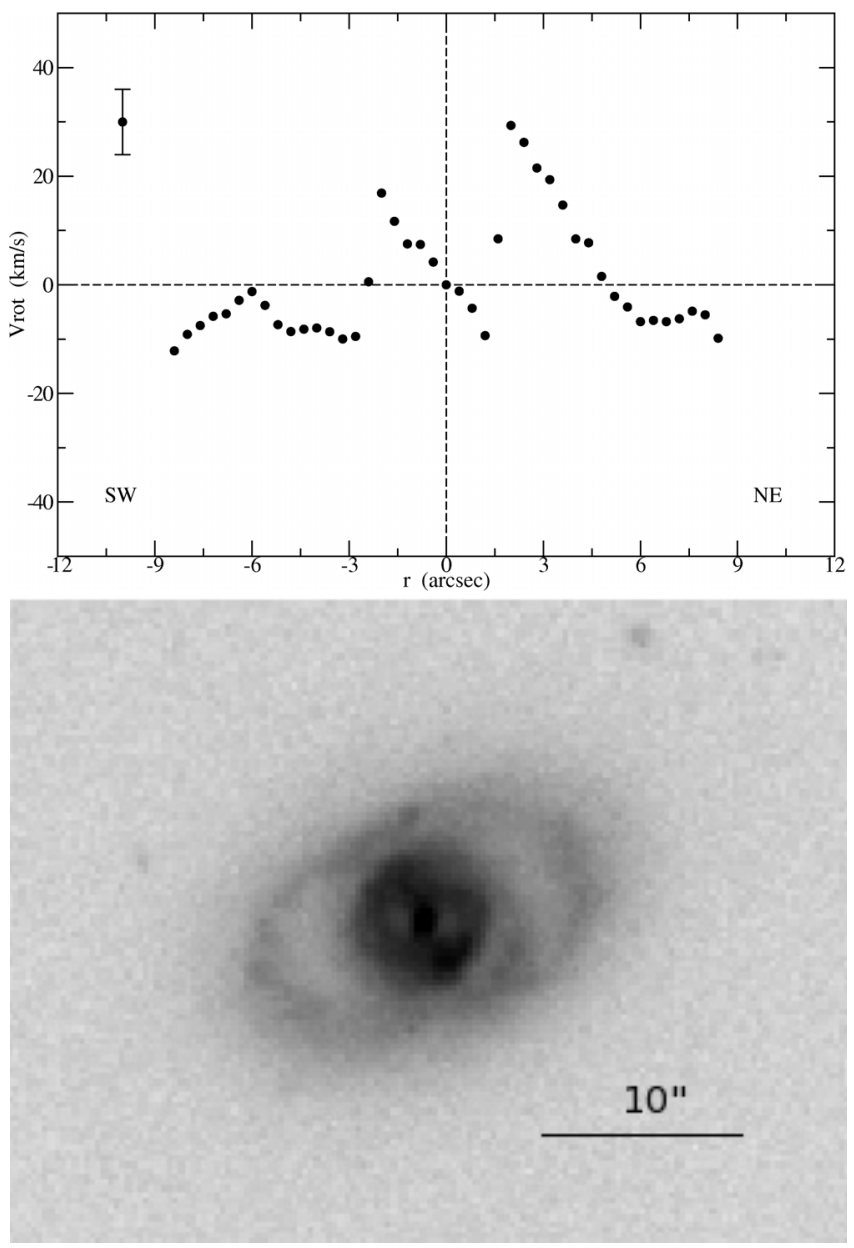

Fig. 9. From top to bottom: a) observed radial-velocity distribution of the nucleus, bulge and ring sections along the ring major axis; $\mathbf{b})$ the $1 .^{\prime} 8 \times 1 .^{\prime} 2 \mathrm{~V}-I$ color map of HRG 54103 (North is up, East is on the right). Some internal structures have been enhanced. The darker the image, the redder the region. The ring itself is bluer than the bulge and nucleus, but its knots are red. Both figures are not to scale.

\subsection{Main source of gas excitation}

In this work, the spectra do not present the complete set of emission lines normally used to classify the activity type in galaxies, as in the classical diagnostic diagrams (see Baldwin et al. 1981; Veilleux \& Osterbrock 1987; Gonçalvez et al. 1998; Kim et al. 1998). In order to overcome this and to decide between the two activity types, we used the diagnostics diagram proposed by Coziol et al. (1999), where we plot the values of the $[\mathrm{N} \mathrm{II}] / \mathrm{H} \alpha$ against $[\mathrm{S} \mathrm{II}] / \mathrm{H} \alpha$ ratios. These two ratios are significantly higher in LINERs and Seyfert2s than in H II regions and Starbursts. This diagram does not allow the distinction between the two AGN types (e.g. Seyferts and LINERs), but regions undergoing photoionization by $\mathrm{O}$ and B stars are clearly separated from AGN ionizing sources. Coziol et al. (1999) established two regions in this diagram where the gas is excited by the two different mechanisms, e.g. AGN and photoionization by stars, which is consistent with the lower limits for the presence of diffuse ionized gas in the halos of edge-on Starbursts as proposed by Lehnert \& Heckman (1996).

If the [N II] excess of emission observed in HRG 54103 the Starburst-AGN behavior - is caused by the addition of other sources of excitation, the intensities of the emission lines should be correlated, that is, they should all increase simultaneously. To
Table 4. Unreddened $\mathrm{H} \alpha$ flux, and nitrogen and sulphur line ratios.

\begin{tabular}{|c|c|c|c|c|c|}
\hline \# & Reg & Pos $(")$ & $\mathrm{H} \alpha\left(\operatorname{erg~cm}{ }^{2} \mathrm{~s}^{-1}\right)$ & {$[\mathrm{N}$ II $] / \mathrm{H} \alpha$} & {$[\mathrm{S} \mathrm{II}] / \mathrm{H} \alpha$} \\
\hline 1 & & -8.0 & $5.81 \times 10^{-13}$ & 0.66 & 0.27 \\
\hline 2 & & -7.6 & $8.05 \times 10^{-13}$ & 0.63 & 0.23 \\
\hline 3 & & -7.2 & $9.61 \times 10^{-13}$ & 0.60 & 0.23 \\
\hline 4 & ExR & -6.8 & $1.20 \times 10^{-12}$ & 0.51 & 0.21 \\
\hline 5 & $\downarrow$ & -6.4 & $1.30 \times 10^{-12}$ & 0.54 & 0.25 \\
\hline 6 & & -6.0 & $1.44 \times 10^{-12}$ & 0.58 & 0.28 \\
\hline 7 & & -5.6 & $1.55 \times 10^{-12}$ & 0.62 & 0.25 \\
\hline 8 & & -5.2 & $1.58 \times 10^{-12}$ & 0.60 & 0.23 \\
\hline 9 & & -4.8 & $1.68 \times 10^{-12}$ & 0.57 & 0.23 \\
\hline 10 & D & -4.4 & $1.71 \times 10^{-12}$ & 0.56 & 0.28 \\
\hline 11 & $\downarrow$ & -4.0 & $1.93 \times 10^{-12}$ & 0.58 & 0.30 \\
\hline 12 & & -3.6 & $2.16 \times 10^{-12}$ & 0.65 & 0.26 \\
\hline 13 & & -3.2 & $1.96 \times 10^{-12}$ & 0.73 & 0.30 \\
\hline 14 & & -2.8 & $1.71 \times 10^{-12}$ & 0.88 & 0.32 \\
\hline 15 & & -2.4 & $1.53 \times 10^{-12}$ & 0.92 & 0.24 \\
\hline 16 & InR & -2.0 & $1.12 \times 10^{-12}$ & 1.15 & 0.37 \\
\hline 17 & B & -1.6 & $9.50 \times 10^{-13}$ & 1.57 & 0.64 \\
\hline 18 & $\downarrow$ & -1.2 & $1.18 \times 10^{-12}$ & 1.98 & 0.56 \\
\hline 19 & & -0.8 & $1.14 \times 10^{-12}$ & 2.10 & 1.12 \\
\hline 20 & $\mathrm{~N}$ & -0.4 & $1.71 \times 10^{-12}$ & 1.97 & 1.13 \\
\hline 21 & $\mathrm{~N}$ & 0.0 & $2.50 \times 10^{-12}$ & 2.02 & 0.67 \\
\hline 22 & $\mathrm{~N}$ & 0.4 & $2.01 \times 10^{-12}$ & 2.12 & 0.81 \\
\hline 23 & & 0.8 & $1.79 \times 10^{-12}$ & 1.93 & 0.96 \\
\hline 24 & $\uparrow$ & 1.2 & $1.90 \times 10^{-12}$ & 1.65 & 0.68 \\
\hline 25 & B & 1.6 & $1.72 \times 10^{-12}$ & 1.46 & 0.43 \\
\hline 26 & InR & 2.0 & $1.43 \times 10^{-12}$ & 1.20 & 0.48 \\
\hline 27 & & 2.4 & $1.87 \times 10^{-12}$ & 0.97 & 0.35 \\
\hline 28 & & 2.8 & $2.34 \times 10^{-12}$ & 0.83 & 0.27 \\
\hline 29 & & 3.2 & $2.57 \times 10^{-12}$ & 0.76 & 0.26 \\
\hline 30 & & 3.6 & $2.60 \times 10^{-12}$ & 0.69 & 0.29 \\
\hline 31 & & 4.0 & $2.64 \times 10^{-12}$ & 0.62 & 0.25 \\
\hline 32 & & 4.4 & $2.36 \times 10^{-12}$ & 0.67 & 0.20 \\
\hline 33 & & 4.8 & $1.81 \times 10^{-12}$ & 0.71 & 0.24 \\
\hline 34 & & 5.2 & $1.58 \times 10^{-12}$ & 0.69 & 0.22 \\
\hline 35 & & 5.6 & $1.34 \times 10^{-12}$ & 0.69 & 0.24 \\
\hline 36 & $\uparrow$ & 6.0 & $1.11 \times 10^{-12}$ & 0.73 & 0.24 \\
\hline 37 & D & 6.4 & $1.01 \times 10^{-12}$ & 0.75 & 0.23 \\
\hline 38 & ExR & 6.8 & $8.19 \times 10^{-13}$ & 0.73 & 0.26 \\
\hline 39 & $\downarrow$ & 7.2 & $7.33 \times 10^{-13}$ & 0.69 & 0.23 \\
\hline
\end{tabular}

Regions in HRG54103: nuclear (N); bulge (B); suggested internal ring (InR); disk (D); and external ring (ExR).

look for such a correlation, we have computed the mean values of $[\mathrm{N} \mathrm{II}] / \mathrm{H} \alpha$ and $[\mathrm{S} \mathrm{II}] / \mathrm{H} \alpha$ ratios. These mean values are given in Table 4 and have been plotted in Fig. 10 against the radial distance in arcsec, respectively. The columns of Table 4 display: (1) the numerical order; (2) the quoted regions in the galaxy (see the description in the tablenote; (3) the arcsec position against the heliocentric systemic velocity center; (4) the unreddened $\mathrm{H} \alpha$ flux; (5) and (6) the $[\mathrm{N} \mathrm{II}] \lambda(6584) / \mathrm{H} \alpha$ and $[\mathrm{S} \mathrm{II}] \lambda(6731) / \mathrm{H} \alpha$ ratios, respectively. It appears that the values of $[\mathrm{N} \mathrm{II}] / \mathrm{H} \alpha$ and $[\mathrm{S} \mathrm{II}] / \mathrm{H} \alpha$ increase as the level of excitation increases (towards the nucleus). This apparent dependence of the emission-line ratios on the level of excitation is consistent with the hypothesis that the emission excess in HRG 54103 is produced by an additional excitation mechanism. On the other hand, the ratios are partly consistent with what is expected if the [N II] and [S II] ratios are sensitive to a variation of abundance when nitrogen is produced by intermediate-mass stars, while sulphur is produced by massive stars (see Torres-Peimbert et al. 1989).

In the case of the HRG 54103 spectroscopic data sample, in the diagram of Fig. 11, the dashed line (Coziol et al. 1999) 

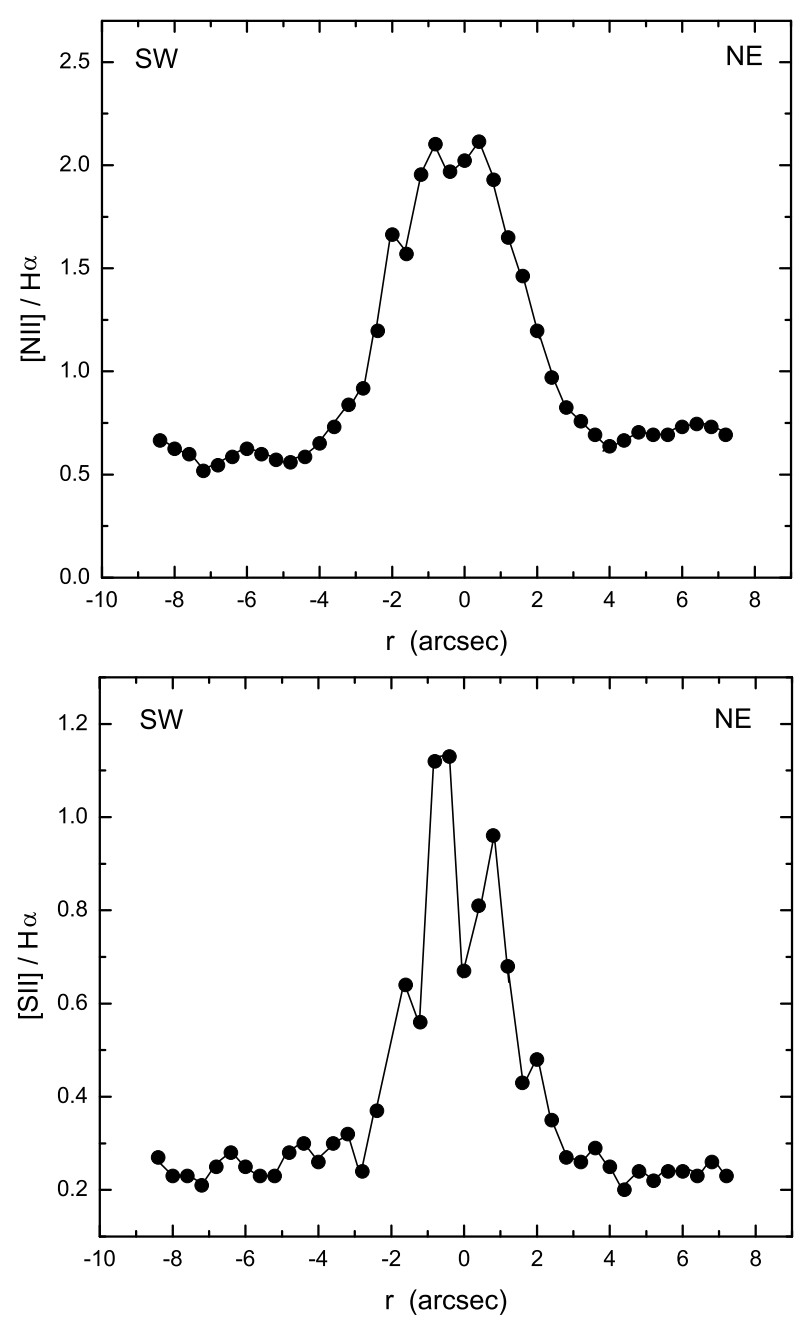

Fig. 10. From top to bottom: a) the $[\mathrm{N}$ II $] \lambda \lambda(6548+6583) / \mathrm{H} \alpha$ ratio; b) the $[\mathrm{S} \mathrm{II}] \lambda \lambda(6548+6583) / \mathrm{H} \alpha$ ratio, both measured along the ring major axis.

clearly shows the separation between regions photoionized by stars, which are located on the ring, and the AGN nuclear contribution. In addition, there is a spreading intermediate distribution with at least five measurements, which lies between the starphotoionization and AGN excitation loci. This intermediate region is probably due to a residual excitation effect from the AGN phenomenon on the contribution of $\mathrm{H}$ II regions. Here, as in a photoionized by stars, there is a positive correlation between the $[\mathrm{N} \mathrm{II}] / \mathrm{H} \alpha$ and $[\mathrm{S} \mathrm{II}] / \mathrm{H} \alpha$ ratios. Most of these intermediate cases lie either inside or at the border of the AGN locus (Coziol et al. 1999).

The $\mathrm{H} \alpha$ emission line has been detected in the spectra of regions between the bulge boundary and the ring. The variation of the values of the $[\mathrm{N} \mathrm{II}] \lambda \lambda(6548+6583) / \mathrm{H} \alpha$ ratio along the ring major axis is shown in Fig. 10. From about $-3.5^{\prime \prime}$ from the center (on the SW direction) to 7" (the end of the wing, ring section on the NE direction), this ratio resembles that of a Seyfert2/LINER. There is a very modest breakpoint at 4 " on the NE direction, which suggests some starburst behavior. This also happens in the SW wing (which contains a knot in the ring) due to the values of the $[\mathrm{N}$ II $] \lambda \lambda(6548+6583) / \mathrm{H} \alpha$ ratio. There is a clear offset from the center of the maximum ratio of $[\mathrm{N} \mathrm{II}] / \mathrm{H} \alpha$, which is double-peaked. As for the two peaks of emission of nitrogen and sulphur, which are almost symmetrically displaced from the

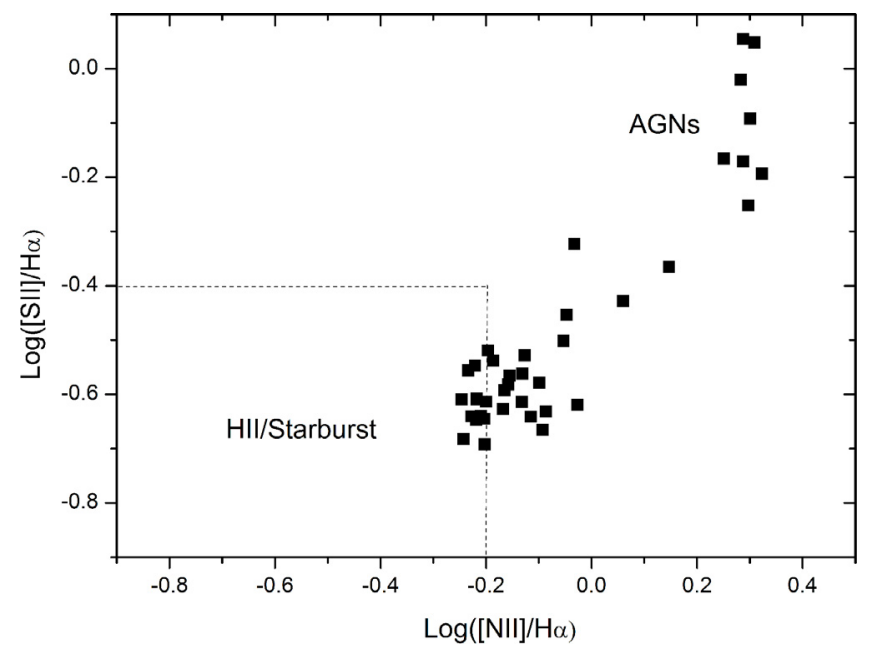

Fig. 11. The $[\mathrm{S} \mathrm{II}] / \mathrm{H} \alpha$ versus $[\mathrm{N} \mathrm{II}] / \mathrm{H} \alpha$ diagnostic diagram.

nucleus of the galaxy $\left(\sim 2^{\prime \prime}\right)$, as seen in Fig. 10, their line intensities are higher than that of the nucleus. This symmetry could be due to either an accretion disk near the central region of the galaxy or a starburst ring whose power source is amplified by the AGN contribution.

Close to the nucleus of HRG 54103 (the upper right data points in Fig. 11), the AGN source dictates the rules of excitation and $[\mathrm{S} \mathrm{II}] / \mathrm{H} \alpha$ increases more abruptly than $[\mathrm{N} \mathrm{II}] / \mathrm{H} \alpha$. This leads to the conclusion that the main source of excitation of the gas in HRG 54103 is an AGN and, therefore, the excess emission of nitrogen observed in this galaxy is due to this powerful source.

\subsection{Kinematics of HRG 54103}

The kinematics of the inner zones of disc galaxies have been shown to be much more complicated than previously thought. Decoupled kinematic cores have been known for some ellipticals, but the kinematics of complex structures are now being seen in disc galaxies, too. There is a variety of dynamical origins for these structures, and they sometimes can be detected separately in the gaseous or in the stellar component, or in both components simultaneously. In spite of gravitational perturbations, some stellar and gaseous components with a misaligned or even opposite angular momentum with respect to its core, conserve some dynamical memory of processes which drive galaxy formation and evolution. In normal galaxies, kinematically-decoupled components ranging from the counter-rotating-decoupled disks of ionized gas are observed in a large fraction of S0 galaxies (Bertola et al. 1992; Kuijken et al. 1996; Plana \& Boulesteix 1996), and orthogonally-rotating stellar cores are found in Sa spirals (Bertola et al. 1999; Sarzi et al. 2000). These characteristics are usually hidden from preliminary morphological inspection and are serendipitously discovered only by detailed kinematics studies (see Bertola \& Corsini 1998 as review).

In this section we propose an internal structure for HRG 54103 based on spectroscopy and direct imagery. Inspecting Fig. 9(a) we realize that there are several kinematical subsystems along the observed slit-signal: (1) a symmetrical core-bulge section at the central region; (2) two isolated data points which suggest the existence of an internal thin ring or a shell external to the bulge; (3) two asymmetrical structures in the SW and NE regions belonging both to the disk and the external visible ring. The velocity distribution of these structures with 
respect to the heliocentric systemic velocity-center suggests that those are kinematically decoupled.

Inspecting Fig. 3, we see that the luminous center of the galaxy (represented by data point \#22 - see Table 4 for numbering of data points) does not coincide with the dynamical center, which is represented by data point \#21, -0.'4 far from \#22. All the remaining discussions will be based on this finding. Moving the center one step, to $-0.4^{\prime \prime}$ in Fig. 9a, the internal rotation curve resembles that of galaxies with kinematically decoupled components with 2 arcsec of the center (Balcells \& González 1998, see also Hau \& Thomson 1994; Rijcke et al. 2004).

There seem to be three rigid rotators with the same angular velocity as suggested by the average inclination of their linear profile, but that exhibit different ranges of tangential velocities. This is in agreement with the fact that the whole galaxy seems to spread to a larger extent on the NE side than on the SW. In Fig. 9(a) their centers lie at $-5.6^{\prime \prime},-0.4^{\prime \prime}$ and $+3.6^{\prime \prime}$, respectively. With respect to the central structure, the one on its left (the smallest one) is approaching while the one on its right is receding from us.

Both the central rotator and the one on the right present the same S-shaped structure around the dynamical mid-point measurement, but the linear segments beyond the discontinuity have the same inclination as the center segment. This suggests that the central part of the galaxy may be constituted by two rigid shells sharing the same rotation, but the external one proceeds slightly behind the inner body. The same would apply for the rotator on the left.

The distance between the three rotators seem to be roughly the same, perhaps as a result of a wave-like or resonance phenomenon. The two data points at $-2.4^{\prime \prime}$ and $+1.6^{\prime \prime}$ (\#16 and \#26, respectively) are symmetrically located with respect to the dynamical center, e.g., \#21, the one on the left receding and the one on the right approaching us. This could be explained by a thin counter-rotating ring.

Taking a closer look at the outer parts of the curve (Fig. 9a), there seems to be an underlying object, perhaps a thin disk, that shows itself as the two horizontal segments at $v \sim-10 \mathrm{~km} \mathrm{~s}^{-1}$ $\left(-5^{\prime \prime} \leq r \leq-3^{\prime \prime}\right.$ and $\left.6^{\prime \prime} \leq r \leq 8^{\prime \prime}\right)$. Should this be true, its left side approaches us at a slightly higher velocity that the portion on the right.

The outermost wings of the velocity curve are measurements made on the external ring, which shares the same behavior of the suggested disk. Careful comparison of the radial-velocity distribution and the $V-I$ color map (see Fig. 9a, b, respectively), for example, allows us to place data points \#16 and \#26 on the edges of the central barred structure, the disk being the apparently "empty" regions on each side of the central rotator and that contains a probable dusty spiral arm which connects with the external ring. In spite of the low resolution spectroscopy, our results suggest a presence of velocity gradients along the disk major axis for HRG 54103, such as some Sa galaxies (e.g. NGC 4698; NGC 2855; see Bertola et al. 1999 and Corsini et al. 2002, respectively).

\section{Conclusions}

In this work, we report observations of the peculiar Ring Galaxy HRG 54103, which was previously classified as a Saturnlike archetype galaxy by Faúndez-Abans \& de Oliveira-Abans (1998a). Our work is based on low resolution spectroscopy and photometric observations in the optical band to highlight some of the morphological and spectral characteristics, and color distribution examined through direct CCD BVRI Kron-Cousins system imagery of this apparently isolated galaxy.

Below is a summary of the results of our work on HRG 54103:

- There are three very low prominence galaxies in the field of HRG 54103 (see Fig. 1): one on the E and two on the SE, the one farthest to the SE having been identified as B011622.67195450.6 (NED). These three galaxies do not have any reported redshifts in the literature (see NED), so it remains to be established whether they are related to HRG 54103 and may be responsible for perturbations which triggered the ring formation.

- The mean integrated surface brightness measurements are in agreement with previous $B_{25^{\prime \prime}}$ (25 mag per square- arcsec ellipse), and $B_{10^{\prime \prime}}$ and $R_{10^{\prime \prime}}$, as referenced in NED (see also The Surface Photometry Catalogue of the ESO-Uppsala Galaxies).

- The color-color diagrams of the bulge and ring have been compared with the archetype star-forming ring galaxy HRG 2302. The individual sections of the color-color index of HRG 54103 are systematically redder than this galaxy's.

- The substructures highlighted in FA98b (see insert in Fig. 1) are redder than the other galaxy components. This may not be a simple scenario. There are three possible causes for this behavior: (a) the presence of older population components of the host galaxy; (b) the substructures are fossil remnants of a past merger, and/or; (c) there is a hidden structure around the bulge - see Fig. 9b, which causes a reddening excess on both the NW and SE sides of HRG 54103.

- The calculated heliocentric systemic velocity is $6483 \pm$ $18 \mathrm{~km} \mathrm{~s}^{-1}(z=0.022)$, which is in agreement with early values in the literature.

- The emission line spectrum suggests the presence of a nuclear Seyfert2/LINER-type object. Emission-line activity is present from 8.4" along the SW direction within 7.2" along the NE direction (see Fig. 10). This behavior is present in both sections of the ring as well as in the fainter sparse material between the bulge and the ring. This scenario is not uncommon in galaxies with rings (see Faúndez-Abans et al. 2009).

- The $[\mathrm{N} \mathrm{II}] \lambda \lambda(6548+6583) / \mathrm{H} \alpha$ ratio profile (Fig. 10) is double-peaked, asymmetrical and off-center with respect to the geometrical center of the intensity profile.

- The emission line measurements along the plane of the ring of HRG 54103 show the presence of activity in the region between the central object and the ring. The $[\mathrm{N} \mathrm{II}] \lambda 6583 / \mathrm{H} \alpha$ ratio behavior can be compared to the ring major axis brightness profile (see Fig. 12), which shows the contribution of the $[\mathrm{NII}] / \mathrm{H} \alpha$ ratio along the nuclear, bulge and ring sections. Based on the results in Table 4, we calculate average $[\mathrm{S} \mathrm{II}] /[\mathrm{N}$ II $]$ ratios of $0.41 \pm 0.09$ and $0.35 \pm 0.03$ on the $\mathrm{SW}$ and NE sides, respectively.

The O-type ring galaxies have a smooth structure, a centrally located nucleus, and no obvious companions. Despite the fact that HRG 54103 is not entirely smooth, it has a centrally located nucleus and no obvious companions, thus, it could still be classified as a prototype of the O-ring galaxy family, in the classification proposed by Few \& Madore (1986). The two bulge satellites, the plume-like appendix, and the disk-ring asymmetry may nonetheless be the relics of the following scenario for the ring formation: in normal galaxies, the standard mechanism to form rings requires the presence of a bar (Binney \& Tremaine 1987; Byrd et al. 1998; Athanassoula et al. 2009); if this is the 


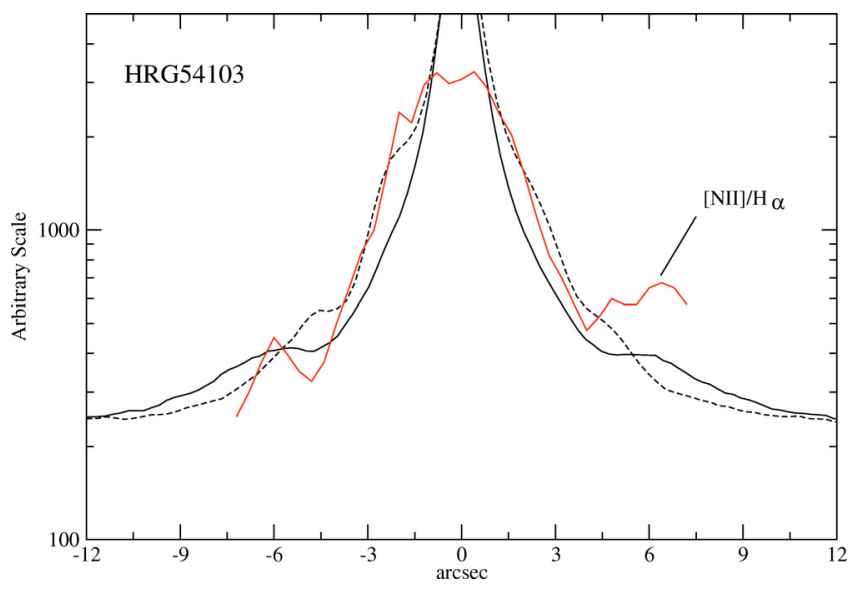

Fig. 12. Same as Fig. 7. The $[\mathrm{N}$ II $] \lambda 6583 / \mathrm{H} \alpha$ ratio (red line) profile could be visually compared with the ring major axis profile (continuous line).

case of HRG 54103, it has probably been dissolved through tidal interaction with a companion, implying that the abovementioned substructures and the disk-ring asymmetry could be the fossil remnants of a possible merger event in the past of this object. A numerical $N$-body/hydrodynamical simulation needs to be used to reconstruct this history and to predict the evolution of the tidal interaction, contributing to an understanding of the morphology of this galaxy.

Acknowledgements. We wish to thank the anonymous referee for the useful comments that helped to improve the paper. This work was partially supported by the Brazilian Ministério da Ciência e Tecnologia (MCT), Laboratório Nacional de Astrofísica, Brazil, grants 381671/2005-4 MCT/CNPq and 680013/20067 MCT/LNA, by the VITAE B-13402/1, by CNPq $473465 / 2004-3$ grant, and UEFS/DFIS/OAA. This publication makes use of data products from the Two Micron All Sky Survey, which is a joint project of the University of Massachusetts and the Infrared Processing and Analysis Center/California Institute of Technology, funded by the National Aeronautics and Space Administration and the National Science Foundation. This research has made use of the NASA/ IPAC Infrared Science Archive, which is operated by the Jet Propulsion Laboratory, California Institute of Technology, under contract with the National Aeronautics and Space Administration.

\section{References}

Appleton, P. N., \& Marston, A. P. 1997, AJ, 113, 201

Appleton, P. N., \& Struck-Marcell, C. 1996, in Fundamentals of Cosmic Physics, 16,111

Appleton, P. N., Gil de Paz, A., Madore, B., et al. 2008, ASP Conf. Ser., 381, 128

Athanassoula, E., Romero-Gómez, M., \& Masdemont, J. J. 2009, MNRAS, 394, 67

Arp, H. C., \& Madore, B. F. 1977, Q. Jl. R. Astron. Soc. 18, 234

Arp, H. C., \& Madore, B. F. 1986, Catalogue of Southern Peculiar Galaxies and Associations, Vol. I, Positions and Descriptions (Toronto: Clarke-Irwin)

Baldwin, J. A., \& Stone, R. P. S. 1984, MNRAS, 206, 241

Baldwin, J. A., Phillips, M. M., \& Terlavich, R. 1981, PASP 93, 5

Balcells, M., \& González, A. C. 1998, ApJ, 505, L109

Bertola, F., \& Corsini, E. M. 1998, in Galaxy Interactions at Low and High Redshift, ed. J. Barnes, \& D. B. Sanders (Dordrecht: Kluwer), IAU Symp., 186,149

Bertola, F., Buson, L. M., \& Zeilinger, W. W. 1992, ApJ, 401, L79
Bertola, F., Corsini, E. M., \& Vega Beltrán, J. C. et al. 1999, ApJ, 519, L127 Bessel, M. 1990. PASP, 102, 1181

Binney, J., \& Tremaine, S. 1987, Galactic Dynamics, (New Jersey: Princeton Univ. Press, Princeton)

Bransford, M. A., Appleton, P. N., Marston, A. P., et al. 1998, AJ, 116, 2757 Burbidge, E. M., \& Burbidge, G. R. 1959, ApJ, 130, 12

Buta, R. 1984, The Structure and Dynamics of Ringed Galaxies, Univ. Tex. Publ. Astron. No. 23, 11, 699

Buta, R. 1995, ApJS, 96, 39

Byrd, G. G., Ousley, D., \& Dalla Piazza, C. 1998, MNRAS, 298, 78

Corsini, E. M., Pizzella, A., \& Bertola, F. 2002, A\&A, 382, 488

Coziol, R., Reyes, R. E. C., Considère, S., Davoust, E., \& Contini, T. 1999, A\&A, 345,733

da Costa, L. N., Willmer, C. N. A., Pellegrini, P. S., et al. 1998, AJ, 116, 1

De Rijcke, S., Dejonghe, H., Zeilinger, W. W., et al. 2004, A\&A, 426, 53

Dominici, T. 2009 (private communication)

Faúndez-Abans, M., \& de Oliveira-Abans, M. 1998a, A\&AS, 129, 357

Faúndez-Abans, M., \& de Oliveira-Abans, M. 1998b, A\&AS, 128, 289 (FA98b)

Faúndez-Abans, M., Hertling, G. P., Ramírez, A. C. 1992, A\&AS, 94, 245

Faúndez-Abans, M., Reshetinikov, V., de Oliveira-Abans, M., et al. 2009, Astron. Lett. 35,25

Fernandes, I. F., de Carvalho, R., Contini, T., et al. 2004, MNRAS, 355, 728

Freeman, K. C., \& de Vaucouleurs, G. 1974, ApJ, 194, 569

Few, J. M. A., \& Madore, B. F. 1986, MNRAS, 222, 673

Fosbury, R. A. E., \& Hawarden, T. G. 1977, MNRAS, 178, 473

Garnier, R., Paturel, G., Petit, C., Martinet, M. C., \& Rousseau, J. 1996, A\&AS, 117,467

Guseva, N. G., Izotov, Y. I. \& Thuan, T. X. 2000, ApJ, 531, 776

González-Delgado, R. M., Perez, E., Diaz, A. I., et al. 1995, ApJ, 439, 604

Gonçalvez, A. C., Véron-Cetty, M.-P., \& Véron, P. 1998, A\&A, 333, 877

Hamuy, M., Walker, A. R., Suntzeff, N. B., et al. 1992, PASP, 104, 533

Hamuy, M., Suntzeff, N. B., Heathcote, S. R., et al. 1994, PASP, 106, 566

Hau, G. K. T., \& Thomson, R. C. 1994, MNRAS, 270, L23

Higdon, J. L. 1995, ApJ, 455, 524

Kennicutt, R. C., Keel, W. C., \& Blaha, C. A. 1989, AJ, 97, 1022

Kennicutt, R. C., Schweizer, F., \& Barnes, J. E. 1996, in Saaa-Fee Advenced

Course 2 - Lecture Notes, Swiss Society for Astrophysics and Astronomy

Kim, D.-C., Veilleux, S., \& Sanders, D. B. 1998, ApJ, 508, 627

Korchagin, V., Mayya, Y. D., \& Vorobyov, E. I. 2001, ApJ, 554, 281

Kuijken, K., Fisher, D., \& Merrifield, M. R. 1996, MNRAS, 283, 543

Landolt, A. U. 1992, AJ, 104, 340

Lauberts, A. 1982, The ESO/Uppsala Survey of the ESO(B) Atlas (Munich: European Southern Observatory)

Lehnert, M. D., \& Heckman, T. M. 1994, ApJ, 426, L27

Lehnert, M. D., \& Heckman, T. M. 1996, ApJ, 462, 651

Lynds, R., \& Toomre, A. 1976, ApJ, 209, 382

Maddox, S. J., Sutherland, W. J., Efstathiou, G., et al. 1990, MNRAS, 243, 692

Marcum, P. M., Appleton, P. N., \& Higdon, J. L. 1992, ApJ, 399, 57

Marston, A. P., \& Appleton, P. N. 1995, AJ, 109, 1002

Mink, D. J., \& Wyatt, W. F. 1995, ASPC, 77, 496

Myrrha, M. L. M., Vaz, L. P. R., Faúndez-Abans, M., de Oliveira-Abans, M., \& Soares, D. S. L. 1999, A\&A, 351, 860 (MVFOS)

Ohyama, Y., Taniguchi, Y., \& Terlevich, R. 1997, ApJ, 480, L9

Origlia, L., Goldader, J. D., Leitherer, C., Schaerer, D., \& Oliva, E. 1999, ApJ, 514, 96

Plana, H., \& Boulesteix, J. 1996, A\&A, 307, 391

Reshetnikov, V. P., Hagen-Thorn, V. A., \& Yakovleva, V. A. 1994, A\&A, 290, 693

Sarzi, M., Corsini, E. M., Pizzella, A., et al. 2000, A\&A, 360, 439

Stasuffer, J. P. 1982, ApJS, 50, 517

Storchi-Bergmann, T. S. 1991, MNRAS, 249, 404

Storchi-Bergmann, T. S., \& Wilson A. S. 1996, In The interplay between massive star formation, the ISM and galaxy evolution, ed. D. Kunth, B. Guiderdoni, N. Heydari-Malayeri, \& T. X. Thuan (France: Éditions Frontières, Gif-surYvette), 613

Theys, J. C., \& Spiegel, E. A. 1976, ApJ, 208, 650

Theys, J. C., \& Spiegel, E. A. 1977, ApJ, 212, 616

Torres-Peimbert, S., Peimbert, M., \& Fierro, J. 1989, ApJ, 345, 186

Veilleux, S., \& Osterbrock, D. E. 1987, ApJS, 63, 295

Vorobyov, E. I., \& Bizyaev, D. 2001, A\&A, 377, 835 